\title{
Pregnancy outcomes in women with gestational diabetes mellitus diagnosed according to the WHO-2013 and WHO- 1999 diagnostic criteria: a multicentre retrospective cohort study
}

Eva A. R. Goedegebure ${ }^{1 \dagger}$, Sarah H. Koning ${ }^{2 *}{ }^{*}$, Klaas Hoogenberg ${ }^{3}$, Fleurisca J. Korteweg ${ }^{4}$, Helen L. Lutgers ${ }^{5}$, Mattheus J. M. Diekman ${ }^{6}$, Eva Stekkinger ${ }^{1}$, Paul P. van den Berg ${ }^{7}$ and Joost J. Zwart ${ }^{1}$

\begin{abstract}
Background: The World Health Organization (WHO) adopted more stringent diagnostic criteria for GDM in 2013, to improve pregnancy outcomes. However, there is no global consensus on these new diagnostic criteria, because of limited evidence. The objective of the study was to evaluate maternal characteristics and pregnancy outcomes in two cohorts in the Netherlands applying different diagnostic criteria for GDM i.e. WHO-2013 and WHO-1999.

Methods: A multicenter retrospective study involving singleton GDM pregnancies in two regions, between 2011 and 2016. Women were diagnosed according to the WHO-2013 criteria in the Deventer region (WHO-2013-cohort) and according to the WHO-1999 criteria in the Groningen region (WHO-1999-cohort). After GDM diagnosis, all women were treated equally based on the national guideline. Maternal characteristics and pregnancy outcomes were compared between the two groups.

Results: In total 1386 women with GDM were included in the study. Women in the WHO-2013-cohort were older and had a higher pre-gestational body mass index. They were diagnosed earlier (24.9 [IQR 23.3-29.0] versus 27.7 [IQR 25.9-30.7] weeks, $p=<0.001)$ and less women were treated with additional insulin therapy (15.6\% versus 43. $4 \%, p=<0.001)$. Rate of spontaneous delivery was higher in the WHO-2013-cohort (73.1\% versus $67.4 \%, p=0.032$ ). The percentage large-for-gestational-age (LGA) neonates (birth weight $>90$ th percentile, corrected for sex, ethnicity, parity, and gestational age) was lower in the WHO-2013- cohort, but not statistical significant (16.5\% versus 18.5\%, $p=0.379$ ). There were no differences between the cohorts regarding stillbirth, birth trauma, low Apgar score, and preeclampsia.
\end{abstract}

Conclusions: Using the new WHO-2013 criteria resulted in an earlier GDM diagnosis, less women needed insulin treatment and more spontaneous deliveries occurred when compared to the cohort diagnosed with WHO-1999 criteria. No differences were found in adverse pregnancy outcomes.

Keywords: Gestational diabetes mellitus, GDM, WHO, Diagnostic criteria, Pregnancy outcomes

\footnotetext{
* Correspondence: s.h.koning@umcg.nl

${ }^{\dagger}$ Equal contributors

${ }^{2}$ Department of Endocrinology, University of Groningen, University Medical

Center Groningen, PO Box 30.001, 9700 RB Groningen, the Netherlands

Full list of author information is available at the end of the article
}

(c) The Author(s). 2018 Open Access This article is distributed under the terms of the Creative Commons Attribution 4.0 International License (http://creativecommons.org/licenses/by/4.0/), which permits unrestricted use, distribution, and reproduction in any medium, provided you give appropriate credit to the original author(s) and the source, provide a link to the Creative Commons license, and indicate if changes were made. The Creative Commons Public Domain Dedication waiver (http://creativecommons.org/publicdomain/zero/1.0/) applies to the data made available in this article, unless otherwise stated. 


\section{Background}

Gestational diabetes mellitus (GDM) is defined as glucose intolerance detected during pregnancy [1]. The prevalence of GDM is increasing and affects between 1 and 14\% of all pregnancies, caused by a global increase in the number of women with obesity around reproductive age and by more stringent diagnostic criteria for GDM [1-4]. Untreated GDM is associated with an increased rate of neonatal and obstetric complications [5-7]. Adverse pregnancy outcomes have been shown to improve with timely diagnosis and treatment of GDM [8].

In 2008, the international prospective Hyperglycemia and Adverse Pregnancy Outcomes (HAPO) study group demonstrated a continuous association between maternal hyperglycaemia and risk of adverse pregnancy outcomes, as birth weight greater than the 90th percentile, caesarean section, premature birth, birth injury, and preeclampsia [9]. Based on these findings and earlier observational studies, the International Association of Diabetes and Pregnancy Study Group (IADPSG) proposed more stringent diagnostic thresholds for GDM [10]. These new diagnostic criteria (fasting plasma glucose level $\geq 5.1 \mathrm{mmol} / \mathrm{l}$ and/or 1 -h plasma glucose level $\geq 10.0 \mathrm{mmol} / \mathrm{l}$ and/or 2 -h plasma glucose level $\geq 8.5 \mathrm{mmol} / \mathrm{l}$ ) have been adopted by the American Diabetes Association in 2010, the World Health Organization (WHO) in 2013, and the International Federation of Gynaecology and Obstetrics in 2015 [1, 11, 12].

However, to date there is no global consensus on these new diagnostic criteria. A recent review on the current European situation showed a lack of consistency on GDM diagnosis [13]. The apparent reluctance to adopt the IADPSG criteria may result from studies showing an increase in prevalence of GDM and thus a higher burden to obstetric healthcare providers [4, 14], but most importantly from scepticism about the clinical benefit of lower diagnostic thresholds $[14,15]$.

Also in the Netherlands there is a debate regarding the diagnostic criteria for GDM. The Dutch Society of Obstetrics and Gynaecology guideline 2010 "Diabetes and Pregnancy" recommends screening for GDM in highrisk women using the 2 -h 75 -g oral glucose tolerance test (OGTT) using the older WHO-1999 criteria, utilizing a fasting blood glucose $\geq 7.0$ and 2-h blood glucose of $\geq 7.8 \mathrm{mmol} / \mathrm{l}[16,17]$. Notwithstanding that, a few hospitals in the Netherlands already implemented the new WHO-2013 thresholds for diagnosis of GDM.

To verify the consequences of implementing these new WHO-2013 thresholds the following question need to be answered: What are the pregnancy outcomes of women diagnosed according the WHO-2013 criteria compared with women diagnosed according the older WHO-1999 criteria?

The objective of the current study was therefore to evaluate the maternal characteristics and obstetric and neonatal outcome in two typical population-based cohorts in the Netherlands which applied the two different diagnostic criteria for GDM i.e. WHO-2013 and WHO-1999.

\section{Methods \\ Study population}

A multicentre, retrospective cohort study was conducted involving three hospitals in the Netherlands (University Medical Center Groningen a tertiary care centre, Martini Hospital Groningen, and Deventer Hospital both secondary care centres). Both regions (Deventer region and Groningen region) are located in the relatively rural north-eastern part of the Netherlands. Part of the data of the Groningen region has been published previously $[18,19]$. All pregnant women with diagnosis of GDM were eligible for inclusion in the study. Women with a twin pregnancy and women with pre-existing diabetes mellitus (DM) were excluded.

This study has been conducted in accordance with the guidelines of the Declaration of Helsinki and Good Clinical Practice. The patient data were retrospectively acquired from hospital records generated during care-as-usual. Statistical analysis was performed requiring patient anonymity in agreement with the ethics committee regulations [20]. According to the Dutch law on Medical Research with Human Subjects, this study has been exempted for approval by the local ethics committees.

\section{Screening, diagnosis and treatment of GDM}

Criteria for screening and diagnosis of GDM are summarized in Fig. 1 [16, 17]. After GDM diagnosis all women were treated based on the national guideline. First, all women received dietary counselling and instructions for self-monitoring of the blood glucose levels (SMBG). According to the guideline, insulin therapy was started if the blood glucose levels were repeatedly above the treatment targets (two blood glucose values above the treatment target at the same day) despite dietary treatment: fasting blood glucose level $>5.3 \mathrm{mmol} / \mathrm{l}$ and/ or either a 1-h postprandial blood glucose level $>7$. $8 \mathrm{mmol} / \mathrm{l}$, or 2-h postprandial blood glucose level $>6$. $7 \mathrm{mmol} / \mathrm{l}$. Options for insulin therapy regimens were: ultra-short-acting insulin, once daily long-acting insulin, or a combination of both (basal-bolus). Metformin was occasionally prescribed in obese women (body mass index $(\mathrm{BMI})>30 \mathrm{~kg} / \mathrm{m}^{2}$ ) in the Deventer hospital (depending on glycaemic control). Based on SMBG women were advised to adjust diet or increase insulinor metformin dose to maintain blood glucose levels within the target range.

Women were seen at the obstetric outpatient clinic regularly and foetal growth was evaluated by ultrasonography at least every 4 weeks. Moreover, all patients were 


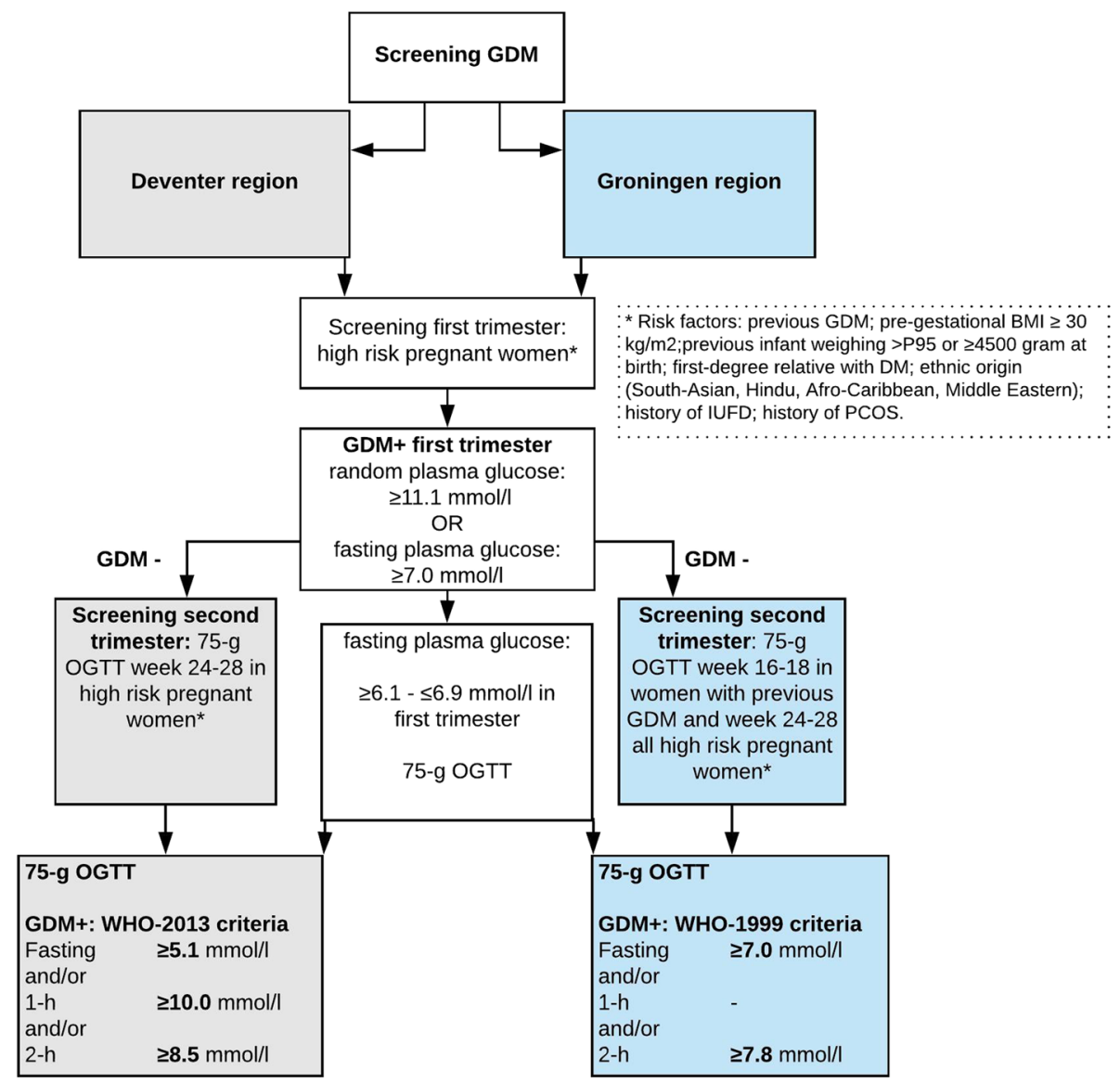

Fig. 1 Screening and diagnosis of gestational diabetes. Abbreviations: GDM, gestational diabetes mellitus; WHO, World Health Organization; OGTT, oral glucose tolerance test; BMI, body mass index; DM; diabetes mellitus; IUFD, intra uterine foetal death; PCOS, polycystic ovary syndrome

discussed every two to three weeks multidisciplinary. Based on similar guidelines in the two regions labour was induced between 38 and 39 weeks of gestation in women on insulin therapy or earlier on indication. In women with a diet, labour was induced between 38 and 40 weeks taking glycaemic control, estimated foetal weight and nonGDM related risk factors into consideration.

\section{Outcomes and definitions}

All electronic medical- and birth records were retrospectively reviewed and data between 2011 and 2016 were included in an anonymised database. Maternal characteristics were age, ethnicity (Caucasian, Asian, African American, Mediterranean or unknown), parity, pre-gestational BMI, risk factors for GDM, hypertensive disorders, results of 75-g OGTT, and treatment details. Chronic hypertension was defined as a systolic blood pressure (SBP) $\geq 140 \mathrm{mmHg}$ and/or a diastolic blood pressure (DBP) $\geq 90 \mathrm{mmHg}$ at booking before 20 weeks of gestation, or the use of blood-pressure lowering drugs before pregnancy.

\section{Obstetric and neonatal outcomes}

Obstetric outcomes collected were induction of labour, mode of delivery (spontaneous vaginal delivery, assisted vaginal delivery (vacuum extraction or forceps), intrapartum caesarean delivery or planned caesarean delivery), gestational age at birth, pregnancy-induced hypertension (PIH) and preeclampsia. PIH was defined as a SBP $\geq 140 \mathrm{mmHg}$ and/or a DBP $\geq 90 \mathrm{mmHg}$, after 20 weeks of gestation in a previously normotensive woman. Preeclampsia was defined ad PIH plus the presence of proteinuria $(\geq 300 \mathrm{mg} / 24-\mathrm{h})$ and also included women who had eclampsia and HELLP syndrome.

Neonatal outcomes were birth weight, large for gestational age (LGA; birth weight $>90$ th percentile, corrected for sex, ethnicity, parity, and gestational age) [21], small for gestational age (SGA; birth weight $<10$ th percentile, corrected for sex, ethnicity, parity, and gestational age) [21], preterm delivery (delivery before 37 weeks of gestation), 5 min Apgar score $<7$, need for respiratory support, still birth/neonatal death, birth trauma (shoulder dystocia, fracture of humerus or clavicle, brachial plexus injury), neonatal hypoglycaemia, 
neonatal hyperbilirubinaemia, and admission to the neonatology department. Of note, neonates with extreme prematurity (delivery before 28 weeks of gestation, $n=$ 3) were excluded prior to the analysis for the variable birth weight. Hyperbilirubinaemia was recorded if the neonate required treatment with phototherapy after birth. Neonatal hypoglycaemia (occurring $>2$-h after birth) was defined as a blood glucose level $<2.6 \mathrm{mmol} / \mathrm{l}$ or treatment with glucose infusion [16]. Neonates born before 32 weeks $(n=2)$ of gestation with neonatal hypoglycaemia were excluded prior to the analysis as hypoglycaemia could well be caused by prematurity. Respiratory support was defined as the need for continuous positive airway pressure after birth or intubation.

\section{Statistical analyses}

Statistical analyses were carried out using statistical package IBM SPSS (version 23.0. Armonk, NY: IBM Corp). Continuous variables are presented as mean \pm standard deviation (SD) or as median and inter quartile range (IQR) according to the normal distribution status. Categorical variables are presented as numbers and frequencies (\%). Appropriate (non)parametric tests were used to compare differences between the groups for continuous variables (independent $t$-test or Mann-Whitney $U$-test in case of skewed distribution) and categorical variables (Chi-square or Fisher's exact test).

To examine the associations between the diagnostic groups and pregnancy outcomes, analyses were performed using logistic regression models in which the ORs and 95\% CIs for the WHO-2013 group were calculated using the WHO-1999 group as reference group. Results were presented as unadjusted models and multivariable-adjusted models, with the multivariableadjusted models adjusted for maternal age, prepregnancy BMI, ethnicity and parity. Only for the variables with sufficient statistical power multivariableadjusted models were performed. The model analysing the association between the diagnostic groups and LGAneonates was adjusted for maternal age and prepregnancy BMI. $P$-value $<0.05$ was considered statistically significant.

\section{Results}

Maternal characteristics are summarized in Table 1. A total of 1386 women with GDM were included in the study, 437 in the WHO-2013-cohort and 949 in the WHO-1999-cohort. In the WHO-2013-cohort, 49.4\% of the women had GDM according to both the WHO-1999 criteria and WHO-2013 criteria. In the WHO-1999cohort, $24.7 \%$ of the GDM women would not have had GDM according to the WHO-2013 criteria.

In total, 1341 women (96.4\%) were diagnosed by OGTT and 45 (3.6\%) women were already diagnosed in first trimester by a random or fasting glucose level. The median fasting glucose level was higher in the WHO2013-cohort and the 2-h glucose level was lower, compared to the WHO-1999-cohort. GDM diagnosis was based on elevated fasting glucose level only in $40.2 \%$ in the WHO-2013-cohort, compared with $0.8 \%$ in the WHO-1999-cohort. GDM was diagnosed based on elevated 2 -h value in $10.9 \%$ in the WHO-2013-cohort and in $95.4 \%$ in the WHO-1999-cohort. Women in the WHO-2013-cohort were diagnosed earlier in pregnancy (24.9 [IQR 23.3-29.0] vs. 27.7 [IQR 25.9-30.7] weeks) and less women had their OGTT performed based on symptoms or signs in third trimester $(15.1 \%$ vs. $28.5 \%)$ instead of screening based on predefined GDM riskfactors. Of the 270 women in the WHO-1999-cohort diagnosed with GDM based on signs suggestive of GDM, $127(47.0 \%)$ retrospectively appeared to have risk factors for GDM. Of these, 12 women tested negative on a first OGTT in the 2nd trimester and 115 women were not screened. In the WHO-2013-cohort $15.6 \%$ of the women received insulin treatment compared with $43.4 \%$ in the WHO-1999-cohort. In the WHO-2013-cohort, 14 (3.2\%) women were treated with metformin.

\section{Obstetric and neonatal outcome}

Table 2 summarizes the obstetric outcomes. In the WHO-2013-cohort there were more spontaneous deliveries $(73.1 \%$ vs. $67.4 \%$, adjusted OR $1.52(1.15-2.01))$, less planned caesarean deliveries $(7.8 \%$ vs. $11.7 \%$, OR 0 . $64(0.43-0.96))$. Median gestational age at birth was higher for women in the WHO-2013-cohort (39.0 vs. 38 . 3 weeks, $p=<0.001$ ) and women in the WHO-2013cohort were less like to have induced labour $(59.3 \%$ vs. $63.9 \%$, adjusted OR $0.76(0.59-0.98)$. There were no differences between the groups with respect to assisted vaginal delivery and intrapartum caesarean delivery. Prevalence of PIH was higher in the WHO-2013-cohort, although no differences were seen between the two groups regarding incidence of preeclampsia.

Table 3 shows the neonatal outcomes. The percentage of LGA neonates (corrected for sex, ethnicity, parity, and gestational age) was lower in the WHO-2013-cohort (16.5\% vs. $18.5 \%$, adjusted OR $0.90(0.66-1.25)$ ), but this was not statistical significant. Birth weight was accordingly higher (3512 vs. 3399 g, $p=<0.001$ ). Neonatal hypoglycaemia was more often diagnosed in offspring of the WHO-2013-cohort (9.6\% vs. $4.2 \%$, adjusted OR 2.48 (1.52-4.05)). There were no significant differences seen between the two groups with respect to neonatal hyperbilirubinaemia, preterm delivery, birth weight in categories, SGA, 5 min Apgar score $<7$, need for respiratory support, birth trauma, still birth/neonatal death, and admission to the neonatology department. 
Table 1 Maternal characteristics of women diagnosed with gestational diabetes mellitus

\begin{tabular}{|c|c|c|c|}
\hline \multirow[b]{2}{*}{ Characteristics } & \multicolumn{3}{|l|}{ Cohort } \\
\hline & WHO-2013 & WHO-1999 & $P$-value* \\
\hline $\mathrm{N}$ & 437 & 949 & \\
\hline Age (years) & $34.7 \pm 5.1$ & $32.1 \pm 5.1$ & $<0.001$ \\
\hline Ethnicity, n (\%) & & & $<0.001$ \\
\hline Caucasian & $357(81.7)$ & $741(78.1)$ & \\
\hline Asian & $9(2.1)$ & $72(7.6)$ & \\
\hline African-American & $2(0.5)$ & $39(4.1)$ & \\
\hline Mediterranean & $64(14.6)$ & $69(7.3)$ & \\
\hline Unknown & $5(1.1)$ & $28(3.0)$ & \\
\hline Parity, n (\%) & & & 0.232 \\
\hline 0 & $158(36.2)$ & $386(40.7)$ & \\
\hline $1-2$ & $242(55.5)$ & 499 (52.6) & \\
\hline$>2$ & $36(8.3)$ & $64(6.7)$ & \\
\hline First degree relative with DM, $\mathrm{n}(\%)$ & $82(18.8)$ & $376(41.1)$ & $<0.001$ \\
\hline History of PCOS, n (\%) & $10(2.3)$ & $50(5.3)$ & 0.011 \\
\hline History of GDM, n (\%) & $44(10.1)$ & $103(10.9)$ & 0.650 \\
\hline Previous infant weighing $\geq 4500 \mathrm{~g}$ at birth, $\mathrm{n}(\%)$ & $42(9.6)$ & $97(10.2)$ & 0.716 \\
\hline History of IUFD, n (\%) & $4(0.9)$ & $20(2.1)$ & 0.113 \\
\hline Pre-gestational BMI $\left(\mathrm{kg} / \mathrm{m}^{2}\right)$ & $29.7[26.0-34.4]$ & $27.7[24.2-31.8]$ & $<0.001$ \\
\hline Pre-gestational BMI, n (\%) & & & $<0.001$ \\
\hline$<25 \mathrm{~kg} / \mathrm{m}^{2}$ & $88(20.8)$ & $291(31.5)$ & \\
\hline $25-29.9 \mathrm{~kg} / \mathrm{m}^{2}$ & $129(30.4)$ & $288(31.2)$ & \\
\hline$\geq 30 \mathrm{~kg} / \mathrm{m}^{2}$ & $207(48.8)$ & $344(37.3)$ & \\
\hline Chronic hypertension, n (\%) & $8(1.8)$ & $43(4.5)$ & 0.013 \\
\hline Indication for OGTT, n (\%) & & & $<0.001$ \\
\hline Screening based on risk factors & $362(82.8)$ & $650(68.5)$ & \\
\hline Diagnostic test based on symptoms/signs & $66(15.1)$ & $270(28.5)$ & \\
\hline Unknown & $9(2.1)$ & $29(3.1)$ & \\
\hline Diagnosis based on OGTT, $\mathrm{n}(\%)^{\ddagger}$ & $422(96.6)$ & $919(96.8)$ & 0.791 \\
\hline Gestational age at time of OGTT (weeks) & $24.9[23.3-29.0]$ & $27.7[25.9-30.7]$ & $<0.001$ \\
\hline Gestational age at time of OGTT screening only (weeks) & 24.4 [22.6-26.9] & $27.3[25.1-28.7]$ & $<0.001$ \\
\hline Gestational age at time of OGTT diagnostic 3rd trimester only (weeks) & $33.1[28.7-35.3]$ & $30.4[27.7-33.6]$ & 0.001 \\
\hline \multicolumn{4}{|l|}{ 75-g OGTT } \\
\hline Fasting glucose level (mmol/l) & $5.3[5.1-5.6]$ & $5.0[4.6-5.5]$ & $<0.001$ \\
\hline 1-h glucose level (mmol/l) & $9.6[8.0-10.5]$ & - & NA \\
\hline 2-h glucose level (mmol/l) & $7.7[6.6-9.0]$ & $8.6[8.1-9.4]$ & $<0.001$ \\
\hline Diagnosis based on elevated fasting glucose level only, n (\%) & $170(40.2)$ & $8(0.9)$ & $<0.001$ \\
\hline Diagnosis based on elevated 2-h glucose level only, n (\%) & $46(10.9)$ & $877(95.4)$ & $<0.001$ \\
\hline Insulin treatment, n (\%) & $68(15.6)$ & $412(43.4)$ & $<0.001$ \\
\hline Metformin treatment, n (\%) & $14(3.2)$ & - & NA \\
\hline
\end{tabular}

Abbreviations: WHO World health Organization, BMI body mass index, DM diabetes mellitus, IUFD intrauterine foetal death, PCOS polycystic ovary syndrome, OGTT oral glucose tolerance test, NA not applicable. Data are expressed as mean $\pm S D$, median [IQR], or proportion of $n(\%)$

*P-values were based on Student's unpaired $t$-test (non-skewed continuous variables), Mann-Whitney U-Test (skewed continuous variables) or chi-square test (categorical variables)

${ }^{*}$ Total number of women diagnosed with a 75-g OGTT. The other women $(n=45)$ were diagnosed with a random or fasting glucose level in first trimester of their pregnancy. Data with respect to first degree relative with DM 35 (3.7\%) (WHO-1999-cohort), BMI 13 (3.0\%) (WHO-2013-cohort) and 26 (2.7\%) (WHO-1999-cohort), gestational age at time of OGT 15 (1.6\%) (WHO-1999-cohort), are missing 
Table 2 Obstetric outcomes of women diagnosed with gestational diabetes mellitus

\begin{tabular}{|c|c|c|c|c|c|}
\hline \multirow[b]{2}{*}{ Outcome variable } & \multicolumn{2}{|l|}{ Cohort } & \multirow[b]{2}{*}{ P-value } & \multirow[b]{2}{*}{$O R^{* *}$} & \multirow[b]{2}{*}{ Adjusted OR** } \\
\hline & WHO-2013 & WHO-1999 & & & \\
\hline $\bar{N}$ & 437 & 949 & & & \\
\hline Induction of labour, n (\%) & $256(59.3)$ & $606(63.9)$ & 0.102 & $0.82(0.65-1.04)$ & $0.76(0.59-0.98)$ \\
\hline \multicolumn{6}{|l|}{ Delivery type, n (\%) } \\
\hline Spontaneous vaginal delivery & $318(73.1)$ & $638(67.4)$ & 0.032 & $1.32(1.02-1.70)$ & $1.52(1.15-2.01)$ \\
\hline Assisted vaginal delivery & $35(8.0)$ & $79(8.3)$ & 0.712 & $0.93(0.61-1.40)$ & NA \\
\hline Intrapartum caesarean delivery & $48(11.0)$ & $121(12.8)$ & 0.365 & $0.85(0.60-1.21)$ & NA \\
\hline Planned caesarean delivery & $34(7.8)$ & $111(11.7)$ & 0.029 & $0.64(0.43-0.96)$ & NA \\
\hline Gestational age at birth (weeks) & $39.0[38.3-39.6]$ & $38.3[38.0-39.0]$ & $<0.001$ & NA & NA \\
\hline Pregnancy-induced hypertension, n (\%) & $50(11.5)$ & $61(6.4)$ & 0.001 & $1.89(1.28-2.80)$ & $1.71(1.11-2.63)$ \\
\hline Preeclampsia, n (\%) & $12(2.8)$ & $30(3.2)$ & 0.683 & $0.87(0.44-1.71)$ & NA \\
\hline
\end{tabular}

Abbreviations: WHO World health Organization, OR odds ratios, NA not applicable. Data are expressed as mean $\pm \mathrm{SD}$, or proportion of $\mathrm{n}$ (\%)

* $P$-values were based on Student's unpaired $t$-test (non-skewed continuous variables), or chi-square test (categorical variables)

**OR, 95\% confidence intervals were derived from logistic regression models using the WHO-1999 group as reference group. Multivariable adjustment included maternal age, pre-gestational body mass index, ethnicity and parity. When the statistical power of a variable was not sufficient or the outcome variable was continuous 'NA' was reported

\section{Discussion}

This multicentre, retrospective cohort study shows the pregnancy outcomes in two cohorts applying different diagnostic criteria for GDM i.e. WHO-2013 and WHO1999. Women in the WHO-2013-cohort had a higher pre-gestational BMI and more often PIH. However, they were diagnosed earlier, less often needed insulin therapy and had a higher percentage of spontaneous deliveries. No other differences in adverse obstetric and neonatal outcomes were seen between the two cohorts.

A number of previous international studies have addressed the effects of introduction of the WHO-2013

Table 3 Neonatal outcomes of women diagnosed with gestational diabetes mellitus

\begin{tabular}{|c|c|c|c|c|c|}
\hline \multirow[b]{2}{*}{ Outcome variable } & \multicolumn{2}{|l|}{ Cohort } & \multirow[b]{2}{*}{$P$-value* } & \multirow[b]{2}{*}{$O R^{* *}$} & \multirow[b]{2}{*}{ Adjusted OR** } \\
\hline & WHO-2013 & WHO-1999 & & & \\
\hline $\bar{N}$ & 437 & 949 & & & \\
\hline Preterm delivery, n (\%) & $27(6.2)$ & $60(6.3)$ & 0.934 & $0.98(0.61-1.57)$ & NA \\
\hline Birth weight (g) & $3512 \pm 459$ & $3399 \pm 532$ & $<0.001$ & NA & NA \\
\hline Birth weight, n (\%) & & & 0.136 & NA & NA \\
\hline Infants $<4000 \mathrm{~g}$ & $384(87.9)$ & $831(87.8)$ & & & \\
\hline Infants $4000-4499 \mathrm{~g}$ & $42(9.6)$ & $104(11.0)$ & & & \\
\hline Infants $\geq 4500 \mathrm{~g}$ & $11(2.5)$ & $11(1.2)$ & & & \\
\hline Large for gestational age, $\mathrm{n}(\%)^{\neq}$ & $72(16.5)$ & $176(18.5)$ & 0.379 & $0.87(0.65-1.18)$ & $0.90(0.66-1.25)$ \\
\hline Small for gestational age, $\mathrm{n}(\%){ }^{\ddagger}$ & $14(3.2)$ & $37(3.9)$ & 0.538 & $0.82(0.44-1.54)$ & NA \\
\hline 5 min Apgar $<7, \mathrm{n}(\%)$ & $7(1.6)$ & $32(3.4)$ & 0.068 & $0.47(0.21-1.08)$ & NA \\
\hline Respiratory support, n (\%) & $14(3.2)$ & $37(3.9)$ & 0.519 & $0.81(0.44-1.52)$ & NA \\
\hline Birth trauma, n (\%) & $15(3.4)$ & $30(3.2)$ & 0.791 & $1.09(0.58-2.05)$ & NA \\
\hline Hypoglycaemia, n (\%) & $42(9.6)$ & $40(4.2)$ & $<0.001$ & $2.41(1.54-3.78)$ & $2.48(1.52-4.05)$ \\
\hline Hyperbilirubinaemia, n (\%) & $4(0.9)$ & $24(2.5)$ & 0.062 & $0.36(0.12-1.03)$ & NA \\
\hline Still birth/neonatal death, $\mathrm{n}(\%)$ & $1(0.2)$ & $2(0.2)$ & 1.000 & NA & NA \\
\hline Admission to the neonatology department, $\mathrm{n}(\%)$ & $54(12.4)$ & $139(14.6)$ & 0.272 & $0.83(0.59-1.16)$ & $0.79(0.55-1.14)$ \\
\hline
\end{tabular}

Abbreviations: WHO World health Organization, OR odds ratios, NA not applicable. Data are expressed as mean \pm SD, or proportion of $\mathrm{n}$ (\%)

${ }^{*} P$-values were based on Student's unpaired $t$-test (non-skewed continuous variables), or chi-square test (categorical variables)

${ }^{*}$ OR, 95\% confidence intervals were derived from logistic regression models using the WHO-1999 group as reference group. Multivariable adjustment included maternal age, pre-gestational body mass index, ethnicity and parity. Large for gestational age was adjusted for maternal age and pre-gestational body mass index. Only for the variables with sufficient statistical power multivariable adjustment was performed. When the statistical power was not sufficient or the outcome variable was continuous 'NA' was reported

${ }^{\ddagger}$ Corrected for sex, ethnicity, parity, and gestational age 
criteria on pregnancy outcomes [22-27]. They retrospectively studied pregnancy outcomes in women previously classified as non-GDM with other diagnostic criteria and newly defined as GDM with the WHO-2013 criteria [22-27]. These studies suggested that women newly diagnosed with the WHO-2013 criteria if untreated were at increased risk for adverse pregnancy outcomes, including $\mathrm{PIH}$, preeclampsia, neonatal intensive care admission, caesarean section, shoulder dystocia, macrosomia and LGA neonates, compared to non-GDM women [22-27]. In contrast to the aforementioned studies, women in our study both diagnosed with WHO2013 or WHO-1999 criteria were treated similarly according to our national guideline. Two comparable studies with regard to treatment and comparison of two diagnostic approaches (Carpenter-Coustan criteria compared with the WHO-2013 criteria) showed that the percentage of LGA neonates was lower in the WHO-2013cohort $[28,29]$. In addition, one study also showed a reduction in caesarean deliveries, PIH, and assisted delivery after implementation of the WHO-2013 criteria [29].

In terms of the likelihood of having an LGA neonate, we found no significant differences between women diagnosed having GDM on the WHO-2013 criteria and women diagnosed having GDM on the WHO-1999 criteria. However, the percentage LGA neonates was lower in the WHO-2013-cohort. The reduction of LGA neonates is an important treatment target in GDM, since LGA is associated with short- and long term complications for the neonate. There are several potential explanations for the lower rates of LGA neonates in the WHO2013-cohort found in our study and others [28, 29]. Firstly, the WHO-2013 criteria included a new group of women: $40.2 \%$ of the women were only diagnosed based on the fasting glucose cut-off value compared to $0.8 \%$ in the WHO-1999-cohort. By applying the more strict WHO-2013 criteria the prevalence of GDM increases, including presumably more mild cases of GDM, resulting in a lower percentage of LGA neonates. Several other studies have demonstrated that implementation of the WHO2013 increases the prevalence of GDM [4, 14]. Moreover, a lower percentage of women in our WHO-2013-cohort (15.6\%) required additional insulin therapy compared with the WHO-1999-cohort (43.4\%).

Secondly, women in the WHO-2013-cohort were screened and diagnosed with GDM earlier (WHO-2013cohort: median $\sim 25$ weeks, WHO-1999-cohort: median $\sim 28$ weeks), so that group had earlier dietary or insulin intervention. More women in the WHO-1999-cohort were diagnosed based on signs suggestive of GDM (e.g. polyhydramnios/foetal macrosomia). Therefore the WHO-1999-cohort may include women with a more advanced stage of GDM leading to higher rates of LGA. Nevertheless, approximately $50 \%$ of all women diagnosed with GDM based on signs suggestive of GDM, retrospectively had a risk factor for GDM that justified 2nd trimester screening in the first place. However, even when we only considered women who were diagnosed based on 2nd trimester screening because of GDM risk factors, gestational age at diagnoses remained different between the groups. The earlier screening and diagnosis of GDM in the WHO-2013-cohort could have led to earlier treatment and therefore to a better outcome. Landon et al. also demonstrated that offering early treatment to women with modest degrees of hyperglycaemia in pregnancy results in reduction of foetal overgrowth [30].

The only obstetric parameters which differed between the two cohorts were the higher incidence of planned caesarean section and induction of labour in the WHO1999-cohort. This may be due to difference in clinical obstetric practice between both regions. But may also be due to differences related to GDM including more estimated macrosomia on ultrasound, worse glycaemic control indicated by significantly more insulin therapy.

An increase in neonatal hypoglycaemia was seen in the WHO-2013-cohort. This can be explained by an active screening policy in all neonates in the hospital that used the WHO-2013 criteria unlike the "WHO-1999 hospitals", that screened neonates by indication. This finding suggests that roughly $50 \%$ neonatal hypoglycaemia might be missed without active screening, potentially leading to long-term adverse outcomes. Moreover, in the WHO2013-cohort a higher percentage of women were diagnosed with PIH. In the WHO-1999-cohort more women were diagnosed with chronic hypertension in first trimester of their pregnancy. This finding suggests that the difference in PIH between the WHO-2013-cohort and WHO-1999-cohort also can be explained by an earlier diagnosis of chronic hypertension in first trimester in the WHO-1999 cohort.

This study gives no information on differences in incidence of GDM between the two diagnostic approaches. In the WHO-2013-cohort, $50.6 \%$ of the women were positive for GDM according the WHO-2013 criteria only and $49.4 \%$ had GDM according to both the WHO-2013 criteria and WHO-1999 criteria. Both cohorts differed in some clinical characteristics: women in the WHO-2013 cohort were older, had a higher pre-gestational BMI and were more often diagnosed on the fasting glucose level compared with the WHO-1999-cohort. These factors are associated with a less favourable metabolic profile. Although the WHO-2013-cohort seemingly consisted of a group of women with milder glucose intolerance, they appeared to have a worse metabolic profile. It seems that the WHO-2013 criteria have a better ability to select women with a worse metabolic profile.

The main strength of this study is that it evaluates the pregnancy outcomes of women with GDM diagnosed by 
the old and new WHO-criteria in a real-life clinical setting. Moreover, after GDM diagnosis all women were treated equally based on the national guideline. Several potential limitations of this study should be noted. First, this study was conducted in three different hospitals in two regions of the Netherlands. It is possible that the study populations and obstetric management between the hospitals were different. One centre is a tertiary care centre and two are larger secondary care centres and this might have led to a selection bias. However, the only important difference between secondary care centres and tertiary care centres in the Netherlands is the referral function for deliveries under 32 weeks of gestational age for neonatal purposes. In all other aspects, population and care is comparable. Secondly, the study was limited by its retrospective study design and this resulted in missing data for some variables in the electronic medical- and birth records. Thirdly, the sample size was limited to find significant differences between the groups for relatively rare pregnancy outcomes, such as birth trauma, still birth/neonatal death, and preeclampsia. Due to the lack of statistical power for some pregnancy outcomes it was not possible to adjust these outcomes for possible confounding factors. Finally, this study gives no information on differences in incidence of GDM between the two diagnostic approaches since the exact number of pregnant women in the two populations is not known. The national guideline advocate targeted testing for GDM, and therefore we do not have data on universal testing.

\section{Conclusions}

In summary, this study demonstrated that application of the WHO-2013 criteria was associated with a reduced need for insulin treatment and more spontaneous deliveries. Although an earlier diagnosis of GDM might contribute to these differences, milder GDM by selection is proposed to play a major role. No differences were found in adverse pregnancy outcomes between the two diagnostic approaches.

This study contributes to the current debate regarding the value of implementation of new WHO-2013 diagnostic criteria for GDM but cannot provide a definitive answer. The data of well conducted population-based randomised studies (and meta-analyses) directly comparing the two diagnostic approaches are necessary to determine whether treatment of women with mild GDM is beneficial and cost-effective. Moreover, there is more information needed whether women with a 2-h glucose value between $\geq 7.8-\leq 8.4 \mathrm{mmol} / \mathrm{l}$ can be safely left untreated.

\section{Abbreviations}

BMI: Body mass index; DBP: Diastolic blood pressure; DM: Diabetes mellitus; GDM: Gestational diabetes mellitus; HAPO: Hyperglycemia and Adverse
Pregnancy Outcomes; IADPSG: International Association of the Diabetes and Pregnancy Study Groups; IQR: Inter quartile range; LGA: Large for gestational age; OGTT: Oral glucose tolerance test; PIH: Pregnancy-induced hypertension; SBP: Systolic blood pressure; SD: Standard deviation; SGA: Small for gestational age; SMBG: Self-monitoring of the blood glucose levels; WHO: World Health Organization

\section{Funding}

Novo Nordisk Netherlands provided an unrestricted research grant for studying the WHO-1999-cohort. The study sponsor was not involved in the designs of the study; the collection, analysis and interpretation of data; writing the report; or the decision to submit the report for publication.

\section{Availability of data and materials}

The datasets generated and/or analysed during the current study are not publicly available. The dataset contains clinical data, which because of the Dutch law for Personal Data Protection and patient confidentiality cannot be shared publicly. Data are available upon request from Prof. Wolffenbuttel. Patients did not sign informed consent to release their data on an individual basis on the internet. For this reason, a research proposal should be filled upon contacting Prof. Wolffenbuttel (bwo@umcg.nl).

\section{Authors' contributions}

EARG, SHK collected and analyzed the data and wrote most of the manuscript under supervision. JJZ, PPB conceived and designed the study and participated in the planning of the project, interpretation of data and writing process and reviewing the manuscript. KH, FJK, HLL, MJMD, and ES made intellectual contributions to the manuscript and have read and approved the final version.

\section{Ethics approval and consent to participate}

This study has been conducted in accordance with the guidelines of the Declaration of Helsinki and Good Clinical Practice. The patient data were retrospectively acquired from hospital records generated during care-as-usual. Statistical analysis was performed requiring patient anonymity in agreement with the ethics committee regulations [20]. According to the Dutch law on Medical Research with Human Subjects, this study has been exempted for approval by the local ethics committees and no informed consent was deemed necessary. The ethical committees of the University Medical Center Groningen and Isala Klinieken Zwolle reviewed the research and also decided that no approval is needed.

\section{Competing interests}

The authors declare that they have no competing interests. Novo Nordisk Netherlands provided an unrestricted research grant for studying the WHO-1999-cohort (SHK and BHRW). The study sponsor was not involved in the designs of the study; the collection, analysis and interpretation of data; writing the report; or the decision to submit the report for publication.

\section{Publisher's Note}

Springer Nature remains neutral with regard to jurisdictional claims in published maps and institutional affiliations.

\section{Author details}

'Department of Obstetrics and Gynaecology, Deventer Hospital, Deventer, the Netherlands. ${ }^{2}$ Department of Endocrinology, University of Groningen, University Medical Center Groningen, PO Box 30.001, 9700 RB Groningen, the Netherlands. ${ }^{3}$ Department of Internal Medicine, Martini Hospital, Groningen, the Netherlands. ${ }^{4}$ Department of Obstetrics and Gynaecology, Martini Hospital, Groningen, the Netherlands. ${ }^{5}$ Department of Internal Medicine, Medical Center Leeuwarden, Leeuwarden, the Netherlands. ${ }^{6}$ Department of Internal Medicine, Deventer Hospital, Deventer, the Netherlands.

7Department of Obstetrics and Gynaecology, University of Groningen,

University Medical Center Groningen, Groningen, the Netherlands.

Received: 8 June 2017 Accepted: 30 April 2018

Published online: 10 May 2018

\section{References}

1. American Diabetes Association. Diagnosis and classification of diabetes mellitus. Diabetes Care. 2014;37(suppl 1):s81-90. 
2. Hunt KJ, Schuller KL. The increasing prevalence of diabetes in pregnancy. Obstet Gynecol Clin N Am. 2007;34:173-99.

3. Ferrara A. Increasing prevalence of gestational diabetes mellitus: a public health perspective. Diabetes Care. 2007;30(suppl 2):s141-6.

4. Moses RG, Morris GJ, Petocz P, San Gil F, Garg D. The impact of potential new diagnostic criteria on the prevalence of gestational diabetes mellitus in Australia. Med J Aust. 2011;194:338.

5. Yang $X$, Hsu-Hage B, Zhang H, Zhang C, Zhang Y, Zhang C. Women with impaired glucose tolerance during pregnancy have significantly poor pregnancy outcomes. Diabetes Care. 2002;25:1619-24.

6. Langer O, Yogev Y, Most O, Xenakis EMJ. Gestational diabetes: the consequences of not treating. Obstet Gynecol. 2005;192:989-97.

7. Sermer M, Naylor CD, Gare DJ, Kenshole AB, Ritchie J, Farine D, et al. Impact of increasing carbohydrate intolerance on maternal-fetal outcomes in 3637 women without gestational diabetes: the Toronto tri-hospital gestational diabetes project. Obstet Gynecol. 1995;173:146-56.

8. Brown J, Alwan NA, West J, Brown S, Mckinlay CJ, Farrar D, Crowther CA. Lifestyle interventions for the treatment of women with gestational diabetes. Cochrane Database Syst Rev. 2017;5:cd01197.

9. Hapo Study Cooperative Research Group. Hyperglycemia and adverse pregnancy outcomes. N Engl J Med. 2008;358:1991-2002.

10. International association of diabetes and pregnancy study groups consensus panel. International association of diabetes and pregnancy study groups recommendations on the diagnosis and classification of hyperglycemia in pregnancy. Diabetes Care. 2010;33:676-82.

11. World Health Organization. Diagnostic criteria and classification of hyperglycemia first detected in pregnancy. 2013.http://apps.who.int/iris/ bitstream/10665/85975/1/who_nmh_mnd_13.2_eng.pdf. Accessed 2 Jun 2017.

12. Hod M, Kapur A, Sacks DA, Hadar E, Agarwal M, Di Renzo GC, et al. The international federation of gynecology and obstetrics (figo) initiative on gestational diabetes mellitus: a pragmatic guide for diagnosis, management, and care. Int J Gynaecol Obstet. 2015;131(suppl 3):s173-211.

13. Benhalima K, Damm P, Van Assche A, Mathieu C, Devlieger R, Mahmood T, et al. Screening for gestational diabetes in europe: where do we stand and how to move forward?: a scientific paper commissioned by the european board \& college of obstetrics and gynaecology (ebcog). Eur J Obstet Gynecol Reprod Biol. 2016;201:192-6.

14. Cundy T, Ackermann E, Ryan EA. Gestational diabetes: new criteria may triple the prevalence but effect on outcomes is unclear. BMJ. 2014;348:g1567.

15. Visser GHA, De Valk HW. Is the evidence strong enough to change the diagnostic criteria for gestational diabetes now? Obstet Gynecol. 2013;208:260-4.

16. The Dutch Society Of Obestetrics And Gynaecology. Diabetes mellitus and pregnancy. Clinical guideline version 2.0. 2010. http://www.nvogdocumenten.nl/index.php?pagina=/richtlijn/item/pagina.php\&richtlijn_id= 863. Accessed 2 Jun 2017.

17. World Health Organization. Definition and classification of diabetes mellitus and its complications. In: Report of a who consultation. Part 1: diagnosis and classification of diabetes mellitus. Geneva: WHO; 1999. Department of noncommunicable disease surveillance.

18. Koning SH, Hoogenberg K, Scheuneman KA, Baas MG, Korteweg FJ, Sollie $\mathrm{KM}$, et al. Neonatal and obstetric outcomes in diet- and insulin-treated women with gestational diabetes mellitus: a retrospective study. BMC Endocr Disord. 2016;16:52.

19. Koning SH, Scheuneman KA, Lutgers H, Korteweg FJ, Van Den Berg G, Sollie KM, et al. Risk stratification for healthcare planning in women with gestational diabetes mellitus. Neth J Med. 2016;74:262-9.

20. University Medical Center Groningen. Research Code University Medical Center Groningen. 2013. https://www.umcg.nl/sitecollectiondocuments/ english/researchcode/umcg-researchcode,\%20basic\%20principles\%202013.pdf. Accessed 2 June 2017

21. Visser GH, Eilers PH, Elferink-Stinkens PM, Merkus HM, Wit JM. New dutch reference curves for birthweight by gestational age. Early Hum Dev. 2009:85:737-44

22. Laafira A, White SW, Griffin CJ, Graham D. Impact of the new iadpsg gestational diabetes diagnostic criteria on pregnancy outcomes in Western Australia. Aust N Z J Obstet Gynaecol. 2016;56:36-41.

23. Benhalima K, Hanssens M, Devlieger R, Verhaeghe J, Mathieu C. Analysis of pregnancy outcomes using the new iadpsg recommendation compared with the carpenter and coustan criteria in an area with a low prevalence of gestational diabetes. Int J Endocrinol. 2013;2013:248121.
24. Meek C, Lewis HB, Patient C, Murphy HR, Simmons D. Diagnosis of gestational diabetes mellitus: falling through the net. Diabetologia. 2015;58:2003-12.

25. O'sullivan E, Avalos G, O'reilly M, Dennedy M, Gaffney G, Dunne F, et al. Atlantic diabetes in pregnancy (dip): the prevalence and outcomes of gestational diabetes mellitus using new diagnostic criteria. Diabetologia. 2011;54:1670-5

26. Lapolla A, Dalfrà M, Ragazzi E, De Cata A, Fedele D. New international association of the diabetes and pregnancy study groups (iadpsg) recommendations for diagnosing gestational diabetes compared with former criteria: a retrospective study on pregnancy outcome. Diabet Med. 2011;28:1074-7.

27. Ethridge JK Jr, Catalano PM, Waters TP. Perinatal outcomes associated with the diagnosis of gestational diabetes made by the international association of the diabetes and pregnancy study groups criteria. Obstet Gynecol. 2014;124:571-8

28. Hung T. The effects of implementing the international association of diabetes and pregnancy study groups criteria for diagnosing gestational diabetes on maternal and neonatal outcomes. PLoS One. 2015;10:e0122261.

29. Duran A, Saenz S, Torrejon MJ, Bordiu E, Del Valle L, Galindo M, et al. Introduction of iadpsg criteria for the screening and diagnosis of gestational diabetes mellitus results in improved pregnancy outcomes at a lower cost in a large cohort of pregnant women: the st. Carlos gestational diabetes study. Diabetes Care. 2014;37:2442-50.

30. Landon MB, Spong CY, Thom E, Carpenter MW, Ramin SM, Casey B, et al. A multicenter, randomized trial of treatment for mild gestational diabetes. N Engl J Med. 2009;361:1339-48.

\section{Ready to submit your research? Choose BMC and benefit from:}

- fast, convenient online submission

- thorough peer review by experienced researchers in your field

- rapid publication on acceptance

- support for research data, including large and complex data types

- gold Open Access which fosters wider collaboration and increased citations

- maximum visibility for your research: over $100 \mathrm{M}$ website views per year

At BMC, research is always in progress.

Learn more biomedcentral.com/submissions 